\title{
The Pho4 transcription factor mediates the response to arsenate and arsenite in Candida albicans
}

\author{
Verónica Urrialde, Daniel Prieto, Jesús Pla and Rebeca Alonso-Monge*
}

Departamento de Microbiología II, Facultad de Farmacia, Universidad Complutense de Madrid, Madrid, Spain

\section{Edited by:}

Helio K. Takahashi, Universidade

Federal de Sao Paulo, Brazil

\section{Reviewed by:}

Leonardo Nimrichter, Federal

University of Rio de Janeiro, Brazil

lan A. Cleary, University of

Tennessee at Martin, USA

Eleonora Kurtenbach, Federal

University of Rio de Janeiro, Brazil

*Correspondence:

Rebeca Alonso-Monge,

Departamento de Microbiología II,

Facultad de Farmacia, Universidad

Complutense de Madrid, Plaza

Ramón y Cajal s/n, E-28040 Madrid,

Spain

e-mail: realonso@farm.ucm.es
Arsenate $(A s(V))$ is the dominant form of the toxic metalloid arsenic (As). Microorganisms have consequently developed mechanisms to detoxify and tolerate this kind of compounds. In the present work, we have explored the arsenate sensing and signaling mechanisms in the pathogenic fungus Candida albicans. Although mutants impaired in the Hog1 or Mkc1-mediated pathways did not show significant sensitivity to this compound, both Hog1 and Mkc1 became phosphorylated upon addition of sodium arsenate to growing cells. Hog1 phosphorylation upon arsenate challenge was shown to be Ssk1-dependent. A screening designed for the identification of transcription factors involved in the arsenate response identified Pho4, a transcription factor of the myc-family, as pho4 mutants were susceptible to As (V). The expression of $\mathrm{PHO} 4$ was shortly induced in the presence of sodium arsenate in a Hog1-independent manner. Pho4 level affects Hog1 phosphorylation upon As (V) challenge, suggesting an indirect relationship between Pho4 activity and signaling in $C$. albicans. Pho4 also mediates the response to arsenite as revealed by the fact that pho4 defective mutants are sensitive to arsenite and Pho4 becomes phosphorylated upon sodium arsenite addition. Arsenite also triggers Hog1 phosphorylation by a process that is, in this case, independent of the Ssk1 kinase. These results indicate that the HOG pathway mediates the response to arsenate and arsenite in C. albicans and that the Pho4 transcription factor can differentiate among As (III), As (V) and $\mathrm{P}_{\mathrm{i}}$, triggering presumably specific responses.

Keywords: Candida albicans, stress response, transcription factor, oxidative stress, arsenate, arsenite, MAPK, signaling

\section{INTRODUCTION}

Arsenic (As) is a metalloid widespread in nature. As occurs with others metalloids, it is toxic and the exposure to it is associated with a variety of diseases in humans, including immunological disorders (reviewed by Flora, 2011). Several compounds containing As are classified as human carcinogens according to the International Agency for Cancer Research. Arsenic can be found in the environment in two main forms: arsenate (As (V)) and arsenite (As (III)). Both prokaryotic and eukaryotic cells have developed mechanisms to take up and detoxify these compounds (Rosen, 2002), the oxidation state being essential for the type of transporter used in this process.

The budding yeast Saccharomyces cerevisiae has been considered as a trustworthy model to unravel the molecular details of metal action and their corresponding detoxification strategies (reviewed by Wysocki and Tamas, 2010). Arsenite enters the cell through the aquaglycerolporin, encoded by FPS1 (Wysocki et al., 2001; Maciaszczyk-Dziubinska et al., 2010), in S. cerevisiae hexose permeases have been also involved in this process (Liu et al., 2004). Fps1 is a bidirectional channel than can also mediate the metalloid's efflux (Bienert et al., 2008). Arsenite is exported from

Abbreviations: As (V), arsenate as $\mathrm{Na}_{2} \mathrm{HAsO}_{4}$ (sodium arsenate); As (III), as $\mathrm{NaAsO}_{2}$ (sodium arsenite); $\mathrm{P}_{i}$, inorganic phosphate. the cytoplasm through the membrane Acr3/Arr3 transporters or transported into the vacuole as a glutathione-conjugated substrate by the Ycf1 ABC-transporter (Wysocki et al., 1997). The expression of these genes is regulated by the transcription factors Arr1 (also called Yap8) and Yap1. Yap1 also prevents oxidative damage in cells exposed to these compounds, by inducing target genes that remove ROS (Reactive Oxygen Species) generated by arsenic compounds (Menezes et al., 2008). Arsenate is a structural analog of inorganic phosphate $\left(\mathrm{P}_{\mathrm{i}}\right)$ and can easily enter the cell through phosphate transporters. Phosphate and arsenate can be imported into the fungal cell by Pho84 and Pho89 (two-high affinity permeases) and Pho87 and Pho90 (two low affinity permeases) (reviewed by Wysocki and Tamas, 2010). In the presence of arsenate, Arr2, an arsenate reductase, reduces arsenate to arsenite which is then removed from the cytoplasm either through Arr3 or Ycf1 (Tamas and Wysocki, 2001; Rosen, 2002).

In order to generate a detoxifying response, cells first have to sense the presence of toxic compounds. Signal transduction pathways mediated by MAP kinases are conserved mechanisms that allow cells sensing and responding to environmental stimuli (Kultz, 1998). These pathways included the MAPK module which is integrated by three protein kinases. These MAP kinases become sequentially activated by phosphorylation and control the expression of proper genes through different transcription factors. 
Signal enters MAPK module through transmembrane proteins, three-component system and/or other kinases. Sensing arsenite in S. cerevisiae is mediated, at least in part, by the HOG pathway (Sotelo and Rodriguez-Gabriel, 2006; Thorsen et al., 2006). The HOG pathway is integrated by two branches: the Sln1-Ypd1Ssk1 that activates Ssk2 and Ssk22 and the Sho1-branch, which comprise Msb2, Sho1, Opy2, Hkr1 that triggers Ste11 phosphorylation. The MAPKKK Ssk2/Ssk22 and Ste11 phosphorylate the MAPKK Pbs2 which activates the MAPK Hog1. Hog1 is phosphorylated in response to arsenite (Sotelo and Rodriguez-Gabriel, 2006) among others stimuli, and induces a cell cycle $\mathrm{G}_{1}$-arrest in a Hog1-dependent manner (Migdal et al., 2008); moreover, it controls the transcription of genes involved in this response. Hog1 also modulates the arsenite uptake by Fps1 (Thorsen et al., 2006).

In addition to the HOG pathway, the cell wall integrity (CWI) pathway mediated by Slt 2 is also involved in the response to arsenate (Matia-Gonzalez and Rodriguez-Gabriel, 2011). CWI pathway mediates the biogenesis of cell wall and incorporates different transmembrane proteins such as Cws1, Cws2, Cws3, Mig2, and Mtl1 which work as receptors (revised by Levin, 2011). These proteins trigger the MAPK module phosphorylation through different mechanisms. The MAPK module is integrated by: Bck1, Mkk1/Mkk2, and Slt2. Slt2 is phosphorylated upon arsenate addition and regulates the expression of several genes, many of them shared with the heat shock response. Arsenite and arsenate therefore trigger different cellular signals and, consequently, different responses in yeast.

Although C. albicans is not an environmental yeast, it was reported to be more resistant to different metal and metalloids than S. cerevisiae (Berdicevsky et al., 1993). The underlying mechanisms of this tolerance remain unknown. Nevertheless, similarly to $S$. cerevisiae, C. albicans also triggers Hog1 phosphorylation upon arsenite exposure and hog1 mutants are more susceptible to this compound (Smith et al., 2004). This suggests that C. albicans and S. cerevisiae share a conserved response to arsenite although the structure of the ortholog pathway is different. In $C$. albicans the HOG pathway has a unique branch comprising a three-component system (Sln1-Ypd1-Ssk1) (Figures 6B, 7) that triggers the MAPK module which includes Ssk2, Pbs2, and Hog1. The transcription factor Skol has been reported to mediate osmotic stress response in a Hogl-depended way (Rauceo et al., 2008). To our knowledge, the signaling pathways involved in response to arsenate have not previously explored in C. albicans. In the present work we have investigated the signaling mechanisms involved in the response to arsenate, the dominant form of arsenic, in the opportunistic pathogen C. albicans and identified the transcription factor Pho4 as a mediator of the response to both arsenate and arsenite in this fungus.

\section{MATERIALS AND METHODS STRAINS AND GROWTH CONDITIONS}

C. albicans strains used in the present work are listed in Table $\mathbf{1 .}$ C. albicans transcription factor mutant collection is deposited and available at the Fungal Genomic Stock Center (http://www.fgsc. net) (Vandeputte et al., 2012). The mutants used in this work are all homozygous null mutants in an Ura + background unless otherwise stated. Yeast strains were grown in YPD medium (1\% yeast extract, $2 \%$ peptone, $2 \%$ glucose), SD (Synthetic Dextrose, $2 \%$ glucose, $0.67 \%$ yeast nitrogen base without amino acids supplemented with synthetic complete dropout URA) or SD low phosphate ( $2 \%$ glucose, $0.57 \%$ yeast nitrogen base with ammonium sulfate, without phosphates, and without sodium chloride (MP Biomedicals) supplemented with synthetic complete dropout URA) supplemented or not with monobasic potassium phosphate $\left(\mathrm{KH}_{2} \mathrm{PO}_{4}\right)$. Growth temperature was $37^{\circ} \mathrm{C}$ unless otherwise indicated. Growth in liquid medium was estimated as the absorbance at $600 \mathrm{~nm}\left(\mathrm{~A}_{600}\right)$. Sensitivity on solid medium to different compounds was tested on plates supplemented with the indicated chemicals at the concentrations indicated in each assay; for these assays, cell number was adjusted to $10^{8}$ cells $/ \mathrm{ml}$ and $1 / 10$ serial cell suspensions were spotted and incubated overnight at $37^{\circ} \mathrm{C}$. Wild type term refers to the corresponding non-mutated isogenic parental strain of the mutations under analysis; as mutants have different backgrounds, specific strain is indicated in the figures. As (V) or arsenate refers to $\mathrm{Na}_{2} \mathrm{HAsO}_{4}$ (disodium hydrogen arsenate or sodium arsenate dibasic). As (III) refers to $\mathrm{NaAsO}_{2}$ or sodium arsenite. The media with arsenic compounds were correctly labeled and, then a specialized company was in charge of its discard.

\section{MOLECULAR BIOLOGY PROCEDURES AND PLASMID CONSTRUCTIONS}

Standard molecular biology procedures were used for all genetic constructions. C. albicans was transformed using the lithium acetate method (Köhler et al., 1997). To reintegrate PHO4 in the $C$. albicans genome, we constructed the pNIM1RPHO4-myc plasmid as follows. The PHO4 ORF was amplified using the primers: o-PHO4Sall-fwd (ACGCGTCGACATGGA CCAGCAAGTTTGGAACCC) and o-PHO4NotI-rev (CCGCGG CCGCCCTTCCTTCCTTTCAACTCC). A $1.99 \mathrm{~Kb}$ fragment was amplified and subcloned in pGEM-T (Promega). Then, the SalINot I fragment was accommodated in the SalI-NotI restriction of the pNIM1R-RFP plasmid (Prieto et al., 2014), generating the pNIM1R-PHO4-myc plasmid. This plasmid carries the PHO4 ORF fused to myc under the control of a repressible tetracycline-regulated promoter. The pNIM1R-PHO4-myc plasmid was digested with KspI and ApaI for its integration in the $A D H 1$ genomic region of the $C$. albicans genome. The strain generated was designed as $\mathrm{PHO}^{\text {reint }}$.

To quantify $\mathrm{PHO} 4$ expression, we have used a C. albicans adapted version of the luciferase from Caribbean Click beetle Pyrophorus plagiophthalamus (CbLUC) (Accession Number KP202872) considering the codon usage of highly expressed genes (Prieto et al., 2014). A 3x HA epitope was placed at the C-terminal of CbLUC sequence as tag (Supplementary Figure 1). We constructed a luciferase plasmid to test promoter activity via the following steps: first, a BamHI-NotI fragment carrying the URA3 marker from pCAHA plasmid (Peter Sudbery, University of Sheffield, UK) was ligated in a pDARD1 (Arana et al., 2007) replacing the SAT1 marker gene and obtaining plasmid pDU0. This plasmid was modified to introduce an additional $K p n I$ site next to the $3^{\prime} A R D 1$ integration sequence generating the plasmid pDUM0. The KpnI restriction site was introduced amplifying the $3^{\prime}$ ARD1 with o-3'-ARD1 Not (GGCCGCGGCCGCAAATAGCGTTCTATTGTCACCC) and 
Table 1 | Strains used in this study.

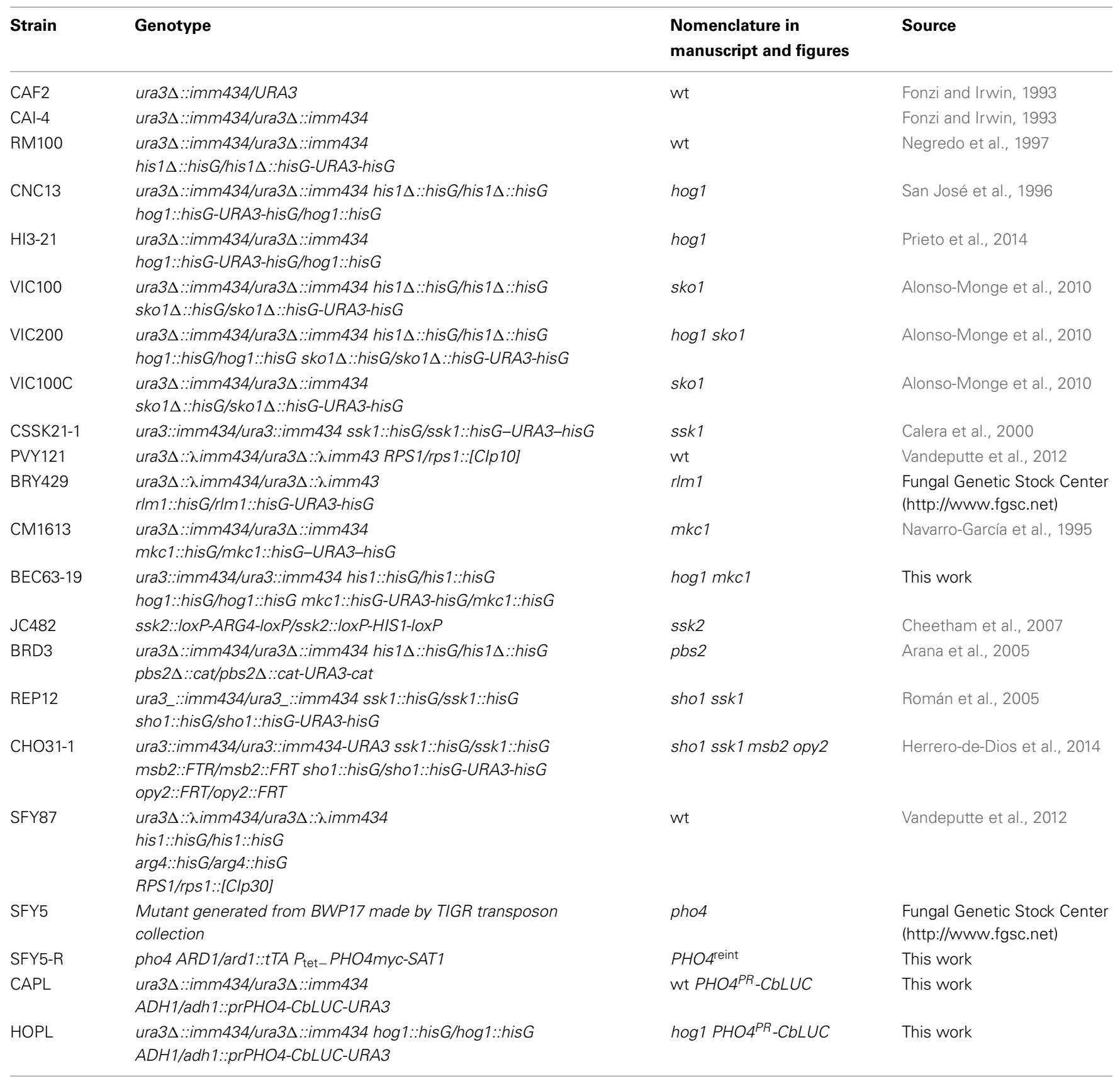

o-3’ARD1 KpnI (GCTGGAGCTCGGTACCTAAAGATAGCAGCG ACAAGGCC).

CbLUC-HA was first cloned into pNIM1R replacing the GFP ORF by digestion with SalI and BglII restriction enzymes. The CbLUC with the ACT1 terminator sequence was then obtained by PCR using the following primers: o-CbLUC XNS fwd (CCGCTCGAGGCTAGCAATAGTCGACGTGAATGGTTAAAAG $A G$ ) and o-ACTTerm $S$ rev (GATACTAGTGGAATGA ATGGGATGAATCATCAAAC). Then, the XhoI-SpeI fragment from the CbLUC-HA PCR was ligated with an XhoI-SpeI pDUM0 plasmid to generate pDUM0-L.
Finally, the 5'upstream region of the $\mathrm{PHO} 4$ ORF was amplified using the following primers: O-PHO4pr fwd (GCTAGCGCTTGACAAAGTAATAAAGGTAAGC) and oPHO4pr rev (GTCGACTGATTTTGCTGAAATCAATGTC). This $1.76 \mathrm{~Kb}$ fragment was subcloned in pGEM-T (Promega) and excised as a NheI-SalI fragment which was then ligated in the NheI-SalI sites of the pDUM0-L. The obtained plasmid, pDUM4-L was digested with KpnI to direct its integration in the $A R D 1$ locus in the C. albicans genome.

The hogl mkc1 mutant was generated by deleting the $\mathrm{MKC1}$ gene in a hog1 mutant (CNC13) following the procedure 
previously described (Navarro-García et al., 1995). The major part of the coding sequence of the $M K C 1$ gene was replaced by the hisG-URA3-hisG cassette.

\section{PROTEIN EXTRACTS AND IMMUNOBLOT ANALYSIS}

Protein extracts were obtained from exponentially growing cultures (O.D. $=1$ ) at $37^{\circ} \mathrm{C}$ in liquid medium after exposure to different compounds. Samples were taken at different time points and processed for western-blot as previously described (Martín et al., 1993). Equal amounts of proteins (assessed by $280 \mathrm{~nm}$ absorbance measurements) were loaded onto gels. Electrophoresis gels were performed at $10 \%$ polyacrylamide while electrophoretic shift was observed at $8 \%$ polyacrylamide gels. Blots were probed with the following antibodies: ScHog1 polyclonal antibody (Santa Cruz Biotechnology) that recognizes CaHog1 protein (43 kDa); Ab-p38-P (Thr180/Tyr182) 28B10 monoclonal antibody (Cell Signaling Technology, Inc.) which detects phosphorylated Hog1, anti-Mkcl antibody (NavarroGarcía et al., 2005) that recognizes Mkcl protein, Ab-phosphop44/42 MAP kinase (Thr202/Tyr204) (Cell Signaling Technology, Inc.) that recognizes $\mathrm{MKcl}(59 \mathrm{kDa})$ and Cek1 $(49 \mathrm{kDa})$ phosphorylated forms and anti-myc Tag (clone 4A6 (Millipore)). Antibodies were used at 1:1000. A fluorescent system (LI-COR Biosciences) that relies on an infrared imaging system with fluorescent secondary antibodies was used to detect and quantify the signal.

\section{LUCIFERASE ASSAY}

Cells carrying the CbLUC under the control of the $\mathrm{PHO} 4$ promoter were grown in liquid medium at $37^{\circ} \mathrm{C}$ and exposed to the compounds under analysis. $10 \mathrm{ml}$ samples were taken, centrifuged $(5000 \mathrm{~g} \times 5 \mathrm{~min}$ ) to obtained cells, washed twice with distilled water, and then resuspended in CbLUC buffer $(50 \mathrm{mM}$ $\mathrm{MgSO}_{4}, 30 \mathrm{mM}$ sodium citrate $\mathrm{pH} 2.5$ ) to a final concentration of $5 \times 10^{7}$ cells $/ \mathrm{ml}$. For each determination, $50 \mu \mathrm{l}$ of cell suspensions were mixed with the same volume of $100 \mathrm{mM} \mathrm{D}$ luciferin in DMSO (SIGMA). Luminescence was measured in an OPTOCOMP Luminometer during $30 \mathrm{~s}$ in integration mode. Results were expressed as fold increase over the control, which was considered the signal detected before substrate addition.

\section{STATISTICAL ANALYSIS}

Statistical differences among two or more groups were calculated using two-way ANOVA. Data are expressed as the mean of at least three experiments \pm standard deviation (SD).

\section{RESULTS}

\section{ARSENATE TRIGgERS Hog1 AND Mkc1 PHOSPHORYLATION}

In order to identify the MAPK pathway involved in arsenate signaling, different C. albicans wild type strains growing exponentially were challenged with $2 \mathrm{mM}$ sodium arsenate and samples were analyzed at different time points. This compound triggered both Hog1 and Mkc1 phosphorylation which was detectable as early as $5 \mathrm{~min}$ after addition (Figure 1A). Hog1 phosphorylation was more prolonged in time than $\mathrm{Mkcl}$ and lasted up to $30 \mathrm{~min}$, while $\mathrm{Mkcl}$ peaked at $5 \mathrm{~min}$ but returned to almost original levels at $30 \mathrm{~min}$. Differences in Hog1 phosphorylation levels

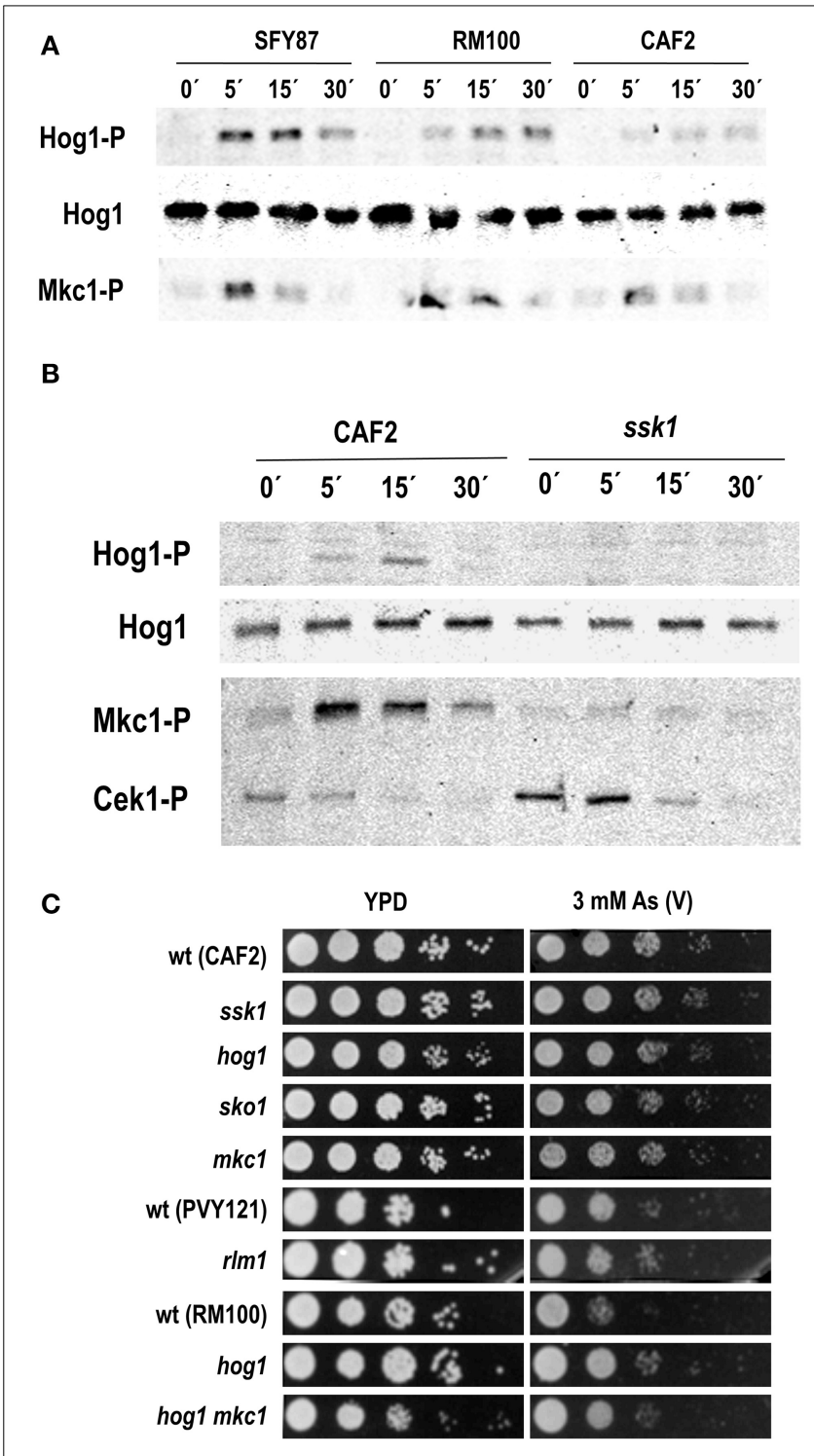

FIGURE 1 | Role of the MAP kinases in the response to arsenate. (A) Three wild type $C$. albicans strains were challenged with $2 \mathrm{mM}$ As $(\mathrm{V})$ and samples collected at indicated time points. (B) CAF2 and ssk1-derived mutant were exposed to $2 \mathrm{mM}$ As $(\mathrm{V})$ and samples taken at the time indicated. MAPKs phosphorylation was detected using specific antibodies. Ab-anti Hog1 was used as loading control. Hog1-P, Cek1-P, and Mkc1-P indicate the phosphorylated form of the MAPKs. Hog1 indicates the total Hog1 protein. (C) Serial dilutions of the indicated C. albicans strains were spotted onto YPD plates (as a control) and YPD supplemented with $3 \mathrm{mM}$ As (V). Mutant strains are shown above their parental strain. Plates were incubated at $37^{\circ} \mathrm{C}$ for $24 \mathrm{~h}$.

were observed among analyzed strains (Figure 1A): phosphorylation was more intense in SFY87 at short times compared to CAF2. Hog1 phosphorylation was fully dependent on Ssk1 as an ssk1 mutant showed no Hog1 phosphorylation (Figure 1B). Mkc1 phosphorylation was also strongly reduced in the ssk 1 mutant, similarly to what has been described to occur in hog1 and pbs2 mutants (Arana et al., 2005) where $\mathrm{Mkcl} \mathrm{H}_{2} \mathrm{O}_{2}$-mediated signaling was highly dependent on the presence of active Hog1. 
The susceptibility to arsenate was tested on solid medium. The lack of Hog1 or Ssk1 did not lead to arsenate sensitivity on YPD plates supplemented with different As (V) concentrations $(1,2$, and $3 \mathrm{mM}$ arsenate were tested) (Figure $\mathbf{1 C}$ and data not shown) and this behavior was not dependent on the temperature of growth (data not shown). Remarkably, RM100 strain displayed an enhanced arsenate susceptibility compare to others wild type strains. Single hog1 (as well as double hog1 mkc1) mutants displayed a susceptibility similar to other backgrounds mutants. The increased susceptibility to arsenate displayed by the RM100 strain was rescued in the presence of $1 \mathrm{M}$ sorbitol, an osmotic stabilizer that reverts some cell wall related phenotypes (data not shown). Additionally, mutants in the transcription factors Sko1 and Rlm1, known targets of the HOG and CWI pathways (respectively), showed no sensitivity to As (V). These results indicate that while the HOG and CWI pathways seem to be involved in the response to arsenate, their impairment do not cause an increased susceptibility to this compound.

\section{SCREENING OF TRANSCRIPTION FACTOR MUTANTS INVOLVED IN THE RESPONSE TO ARSENATE}

With the aim of identifying the putative transcription factor involved in the resistance/tolerance to As (V), a 241 transcription factor knock out library from C. albicans was analyzed (available at Fungal Genetic Stock Center (http://www.fgsc.net)). Ten-fold serial dilutions of each strain were plated onto YPD plates supplemented with $1 \mathrm{mM}$ or $2 \mathrm{mM}$ arsenate. Five out of 241 mutants displayed susceptibility to arsenate compared to their isogenic parental strains. These genes are summarized in Table 2 while arsenate sensitivity is shown in Supplementary Figure 2. Among the mutants that displayed sensitivity to arsenate we identified PHO4, a basic helix-loop-helix (bHLH) transcription factor of the myc-family involved in the response to phosphate limitation.
Since arsenate is structurally similar to phosphate, the pho4 mutant was selected to perform further analyses.

\section{IN SILICO ANALYSIS OF C. ALBICANS Pho4}

The PHO4 ortholog gene in S. cerevisiae encodes a basic helixloop-helix (bHLH) transcription factor of the myc-family. This transcription factor activates transcription cooperatively with Pho2p in response to phosphate limitation being regulated by phosphorylation at multiple sites (Komeili and O'Shea, 1999). When environmental phosphate concentration is high, Pho4 is hyper-phosphorylated by Pho80-Pho85 complex, which switches off $\mathrm{PHO}$ pathway and excludes Pho4 from the nucleus. Excess of phosphate is stored in the form of poly phosphate in vacuoles (recently reviewed by Tomar and Sinha, 2014). When the environmental phosphate concentration is low, Pho81 represses the Pho80-Pho85 complex, leading to Pho4 hypo-phosphorylation. Then, Pho4 remains in the nucleus switching on the pathway and inducing the expression of high-affinity transporters (Pho84 and Pho89) and secretory phosphatases (Pho5, Pho11, and Pho12).

$\mathrm{ScPho} 4$ is a 312 aminoacid (aa) protein that becomes phosphorylated by the Pho80-Pho85 kinase complex on five Ser-Pro (SP) dipeptides (SP1, SP2, SP3, SP4, and SP6) (Komeili and O'Shea, 1999). Phosphorylation of SP2 and SP3 leads to the nuclear export of Pho4. Phosphorylation at SP4 site inhibits its nuclear import. Phosphorylation at SP6 blocks the interaction of Pho4 with the transcription factor Pho2. The function of SP1 phosphorylation has not been elucidated. Subsequently, unphosphorylated Pho4 is accumulated in the nucleus and controls the expression of phosphate-responsive genes. CaPHO4 encodes a 659 aa length protein. The comparison between ScPho4 and CaPho4 shows that both proteins conserve a DNA binding motif (data base Pfam) at the C-terminal of the sequence (251-310 in S. cerevisiae, 595-648 in C. albicans (Supplementary Figure 3). Eight putative phosphorylatable Ser-Pro dipeptides can be detected in

Table 2 | Transcription factors whose deletion results in enhanced susceptibility to arsenate.

\begin{tabular}{|c|c|c|c|}
\hline Gene name & Description CGD & Strain & Assembly 21 ORF number \\
\hline $\mathrm{PHO4}$ & $\begin{array}{l}\text { bHLH transcription factor of the myc-family; required for growth } \\
\text { in medium lacking phosphate and for resistance to copper and } \\
\text { Phloxine } \mathrm{B} \text {; induced by Mnl1 under weak acid stress }\end{array}$ & SFY5 & orf19.1253 \\
\hline ORF19.2260 & Putative transcription factor with zinc finger DNA-binding motif & CJN854 & orf19.2260 \\
\hline CAP1 & $\begin{array}{l}\text { AP-1 bZIP transcription factor; apoptotic, oxidative stress } \\
\text { response/Resistance, multidrug resistance; nuclear in oxidative } \\
\text { stress; complements } S \text {. cerevisiae yap } 1 \text { mutant; oralpharyngeal } \\
\text { candidasis-, human neutrophil, Spider biofilm induce }\end{array}$ & CJN608 & orf19.1623 \\
\hline
\end{tabular}

WOR3 Transcription factor; modulator of white-opaque switch; induced

CJN432 orf19.467

in opaque cells; promoter bound by Wor1; overexpression at

$25^{\circ}$ shifts cells to opaque state; deletion stabilizes opaque cells

at higher temperatures; Spider biofilm induced 
the CaPho4 sequence. Only one out of eight putative phosphorylation sites do concur when $\mathrm{ScPho} 4$ and $\mathrm{CaPho} 4$ are aligned. The presence of a Ser-Pro dipeptide together with other putative phosphorylation residues suggests that $\mathrm{CaPho} 4$ could be regulated by phosphorylation similarly to S. cerevisiae Pho4.

\section{Pho4 LEVELS INFLUENCE Hog1 PHOSPHORYLATION ON THE PRESENCE AND ABSENCE OF STRESS}

To allow Pho4 detection, the PHO4 gene was epitope-tagged with myc and cloned in pNIM1R plasmid. This plasmid carries a repressible version of the doxycycline $O P 4$ promoter. The construction was integrated at the $A D H 1$ genomic region in the pho4 mutant. The generated strain was named pho4 reintegrant or $\mathrm{PHO}^{\text {reint }}$. It must be remarked that in this strain, PHO4 expression levels are constitutive and therefore, different to those derived from physiological levels. The reintegrant strain was included in the analyses and the role of Pho4 in MAPK phosphorylation was analyzed in the presence of As (V). The lack of PHO4 did not impair Hog1 phosphorylation but rather, it prolonged it in time. Pho4 reintegration increased Hog1 phosphorylation under basal conditions (Figure 2A), that is, in the absence of arsenate. These data suggest that Pho4 levels do affect the response of Hog1 to arsenate. Mkcl phosphorylation remained essentially unaffected by Pho4 (Figure 2A lower panel). We also checked the role of osmotic and oxidative stress (known to trigger Hogl phosphorylation) in pho4 mutant strains. Interestingly, the lack of $\mathrm{Pho} 4$ diminishes Hog1 phosphorylation upon $1.5 \mathrm{M} \mathrm{NaCl}$ or $10 \mathrm{mM} \mathrm{H}_{2} \mathrm{O}_{2}$ (Figures 2B,C). Conversely, Pho4 over-expression increased basal Hog1 phosphorylation and these cells were still able to increase Hog1 phosphorylation upon the addition of $1.5 \mathrm{M} \mathrm{NaCl}$ (Figure $2 \mathrm{~B}$ ) or $10 \mathrm{mM}$ hydrogen peroxide (Figure 2C). These data indicated that expression of Pho4 is important for Hogl signaling and supports a relationship between the HOG pathway and phosphate metabolism.

\section{Pho4 IS INVOLVED IN THE RESPONSE TO ARSENATE}

The susceptibility to arsenate was tested between the parental wild type (SFY87) and both pho4 and $\mathrm{PHO} 4^{\text {reint }}$ mutants (Figure 3A). A $\mathrm{PHO}^{\text {reint }}$ behaved as the wild type strain on As (V) supplemented plates, indicating that the $\mathrm{PHO} 4$-myc fusion was functional and that the susceptibility to arsenate was Pho4-dependent. The effect of As (V) on Pho4 was also analyzed. For this purpose, a pho4 mutant carrying the ectopic PHO4-myc allele was challenged with $2 \mathrm{mM}$ As (V) and samples were collected at different time points; Pho4-myc was detected using anti-myc antibodies (Figure 3B). A slight electrophoretic shift was detected within 5 min after As (V) challenge, indicating an increase in the molecular weight.

Pho4 expression was also quantified using a C. albicans codon optimized version of the Click beetle luciferase as gene reporter. For this purpose, a CAI4 wild type strain carrying the $P H O 4^{P R}$ CbLUC construction growing exponentially in YPD was challenged with $2 \mathrm{mM}$ As (V). Pho4 expression was transiently and significantly induced $15 \mathrm{~min}$ after As (V) addition; this increase was independent on the presence of Hog1 as a hog1 mutant displayed a similar pattern of Pho4 expression (Figure 3C). Collectively, these data indicate that As (V) induced both the

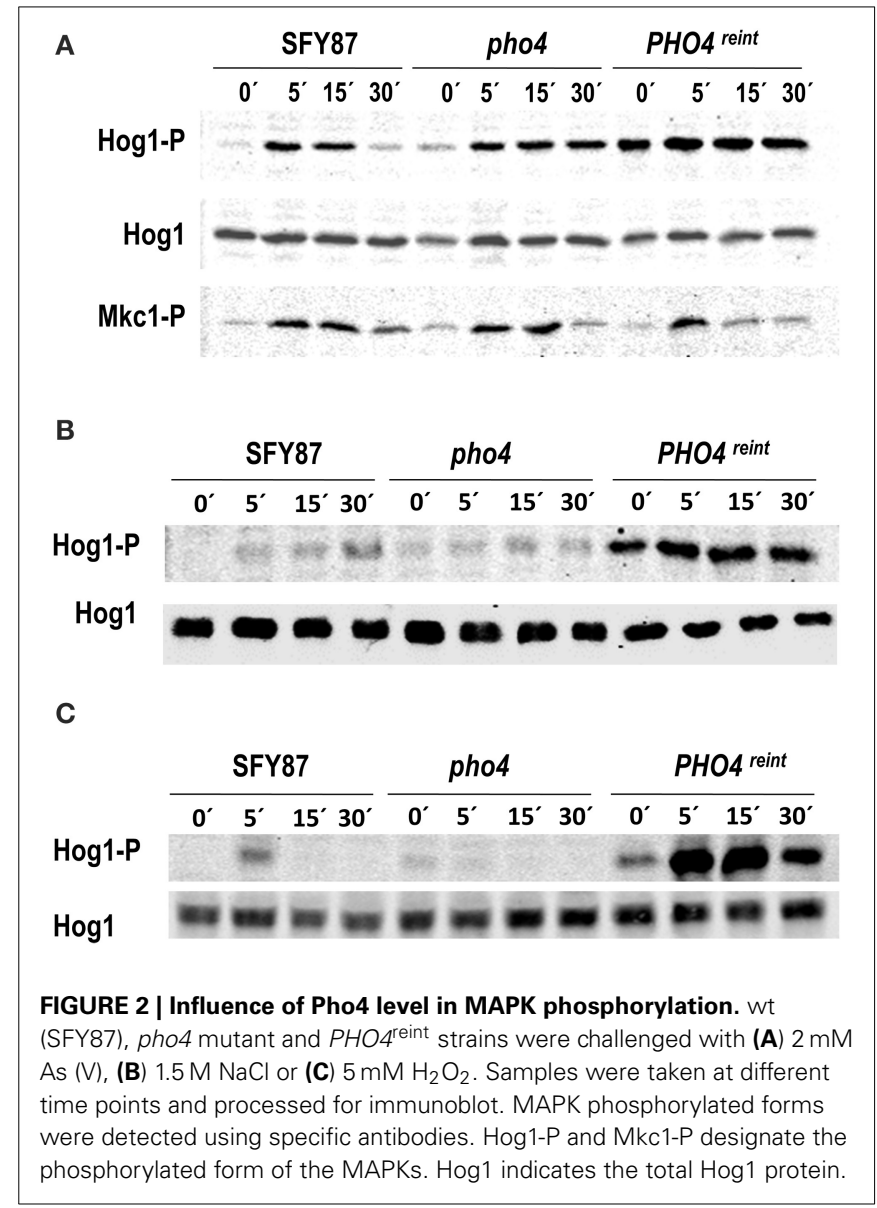

expression of Pho4 expression and an electrophoretic delay, presumably due to Pho4 phosphorylation, while the lack of Pho4 rendered mutant sensitive to As $(\mathrm{V})$. We therefore conclude that Pho4 mediates the response to arsenate.

\section{Pho4 DIFFERENTIATES BETWEen ARSENATE AND PHOSPHATE}

The growth defect of pho4 mutants on low phosphate SD medium plates was further analyzed. A drop test was performed comparing the growth on SD medium and SD medium prepared with a low phosphate Yeast Nitrogen Base (see Materials and Methods). pho4 mutants displayed growth defects on low phosphate SD medium, indicating that Pho4 mediates adaptation to a low phosphate environment. As expected, the $\mathrm{PHO}^{\text {reint }}$ was able to grow as the wild type strain (Figure 4A).

In low phosphate medium, S. cerevisiae Pho4 remains unphosphorylated but becomes phosphorylated when the phosphate concentration increases (reviewed by Tomar and Sinha, 2014). To analyze the behavior of Pho4 in C. albicans, the PHO4 ${ }^{\text {reint }}$ strain was grown in SD low phosphate and shifted to SD supplemented with $2 \mathrm{mM}$ inorganic phosphate $\left(\mathrm{P}_{\mathrm{i}}\right)$. Samples were taken at different time points and changes in the electrophoretic mobility analyzed (Figure 4B). A delay in mobility was observed after 5 min that lasted at least 15 min after the medium shift, suggesting that $\mathrm{Pho} 4$ became phosphorylated upon the increase of $\mathrm{P}_{\mathrm{i}}$ concentration. 


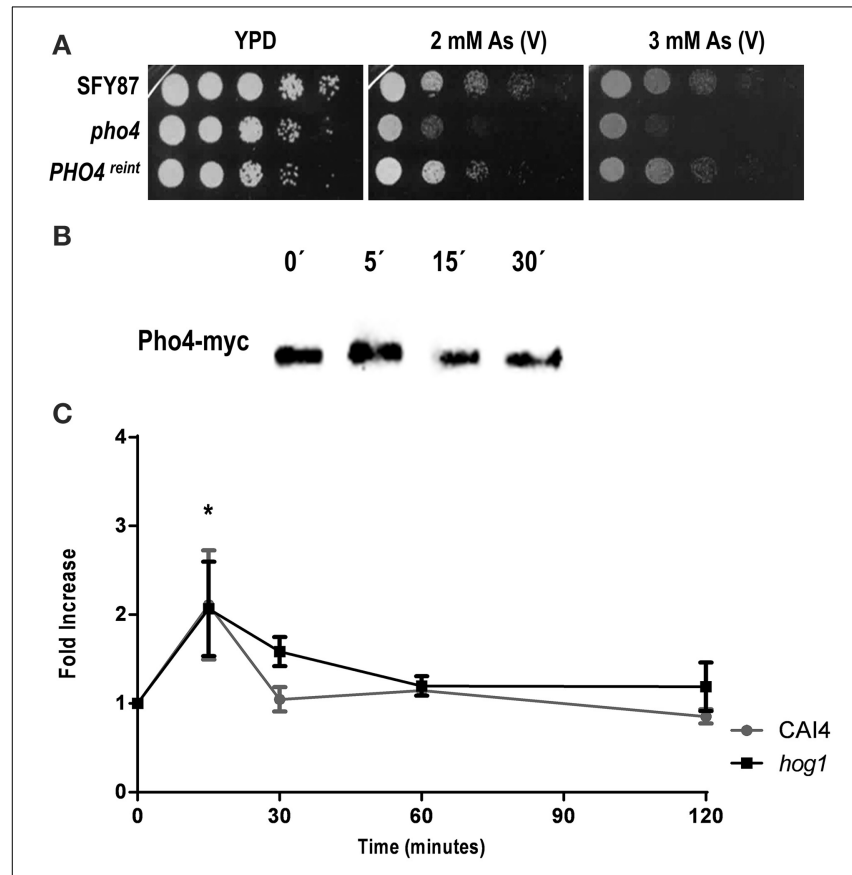

FIGURE 3 | Pho4 mediates the response to arsenate. (A) Ten-folds cell dilutions of the indicated strains were spotted on YPD plates supplemented with 2 and $3 \mathrm{mM}$ As (V). Plates were incubated at $37^{\circ} \mathrm{C}$ for $24 \mathrm{~h}$. (B) Re-integrant PHO4-myc strain was challenged with $2 \mathrm{mM}$ As (V) for different time points and samples collected and processed for immunoblot. Pho4-myc was detected using anti-myc antibodies. (C) Pho4 expression was quantified using CbLUC as gene reporter. CAI4 and hog1 mutant strains carrying $P H O 4^{P R}$-CbLUC growing exponentially in YPD were challenged with $2 \mathrm{mM}$ As $(\mathrm{V})$ and samples were taken and processed for luminescence measurement. $\mathrm{PHO} 4$ expression is expressed as fold induction related to YPD basal expression. Data represent the averages for three independent experiments. Error bars represent the standard deviation (SD). $* P \leq 0.05$.

We also asked if $\mathrm{P}_{\mathrm{i}}$ and As $(\mathrm{V})$ were sensed similarly by the MAPK network due to their structural similarity. Cultures grown in SD low phosphate medium were split and challenged with either $2 \mathrm{mM} \mathrm{P}_{\mathrm{i}}$ or $2 \mathrm{mM}$ As (V). Phosphate triggered Hogl and Cek1 phosphorylation while no phosphorylation was detected at all in response to arsenate (Figure 4C). These data indicate that $C$. albicans differentiates between arsenate and phosphate. Remarkably, arsenate is not sensed by MAP kinases in these experimental conditions. This effect was dependent on phosphate availability: when a similar experiment was performed starting from medium with phosphate (SD), the addition of $\mathrm{P}_{\mathrm{i}}(2 \mathrm{mM})$ or As (V) (2 mM) showed a complete different behavior: while $\mathrm{P}_{\mathrm{i}}$ induced a slight activation of Hog1, As (V) triggered Hog1 and Mkcl similarly to the results reported above (Figure $\mathbf{1}$ and Supplementary Figure 4).

The influence of phosphate in Pho4 expression was analyzed. For this purpose, we used cells from a CAI4 strain carrying the $\mathrm{PHO}^{P R}{ }^{\mathrm{R}}$-CbLUC construction growing on SD low phosphate supplemented with $2 \mathrm{mM} \mathrm{P}$. Cells were collected, washed twice with SD low phosphate and released in different media: (1) SD low phosphate, (2) SD low phosphate plus $2 \mathrm{mM} \mathrm{P}_{\mathrm{i}}$, (3) SD low phosphate plus $2 \mathrm{mM}$ As (V) or (4) SD low phosphate plus $2 \mathrm{mM}$

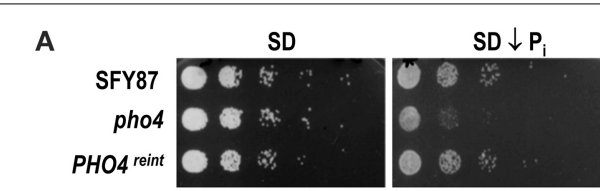

B

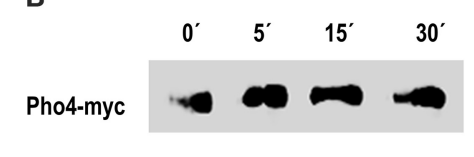

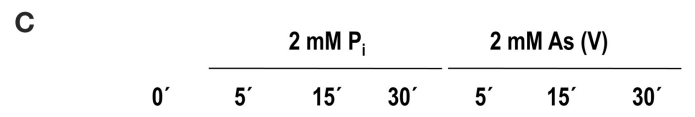
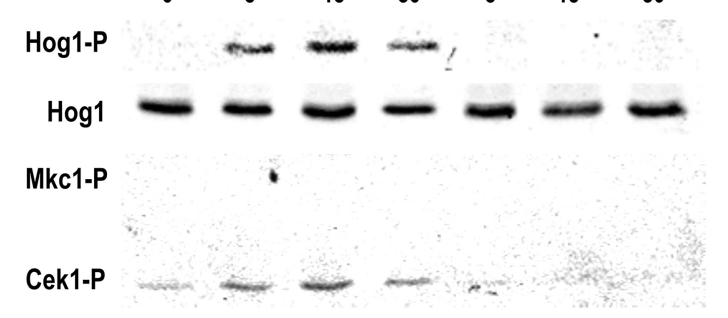

D

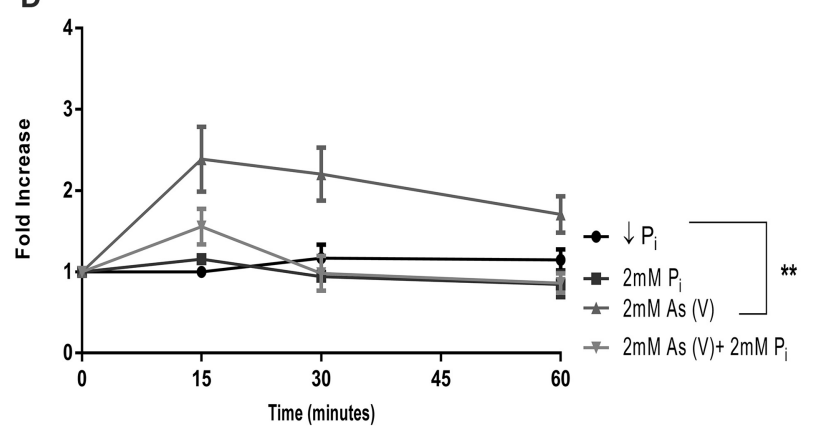

FIGURE 4 | C. albicans differentiates between phosphate and arsenate. (A) Growth of the indicated strains on SD and SD low phosphate plates. Plates were incubated at $37^{\circ} \mathrm{C}$ for $24 \mathrm{~h}$. (B) $\mathrm{PHO} 4^{\text {reint }}$ strain incubated in SD low phosphate overnight at $37^{\circ} \mathrm{C}$ was shifted to $\mathrm{SD}$ and samples were taken at different times points. Then, samples were processed for western-blot and Pho4-myc was detected using specific anti-myc antibodies. (C) The wild type strain CAF2 growing in SD low phosphate medium was challenged with $2 \mathrm{mM} \mathrm{P}_{\mathrm{i}}$ or $2 \mathrm{mM}$ As (V). Samples collected at different time points and MAPKs phosphorylation detected using specific antibodies. (D) The CAF2 strain carrying the $P H O 4^{P R}$-CbLUC grown exponentially in SD low phosphate supplemented with $2 \mathrm{mM} \mathrm{P}$ were washed twice and shift to SD low phosphate, SD low phosphate supplemented with $2 \mathrm{mM} \mathrm{P}_{\mathrm{i}}, 2 \mathrm{mM}$ As $(\mathrm{V})$ or $2 \mathrm{mM} \mathrm{P}_{\mathrm{i}}$ plus $2 \mathrm{mM}$ As $(\mathrm{V})$. Samples were taken at time indicated and luminescence quantified. The graph represents the mean of four independent experiments. Error bars represent the standard deviation (SD). ${ }^{* *} p \leq 0.01$.

As $(\mathrm{V})$ and $2 \mathrm{mM} \mathrm{P}_{\mathrm{i}}$. Induction of $\mathrm{Pho} 4$ expression was followed on time (Figure $4 \mathrm{D}$ ). The shift from low to $2 \mathrm{mM} \mathrm{P}_{\mathrm{i}}$ supplemented medium did not have effect on Pho4 expression, at least at the time points analyzed. However, $2 \mathrm{mM}$ As (V) led to a 2.5 -fold induction of Pho4 expression at $15 \mathrm{~min}$ and decreased slightly remaining higher compare to the others conditions tested. When C. albicans cells were released on SD medium supplemented with equal amounts of arsenate and phosphate, the expression of Pho4 
increased almost twice (similarly to the expression observed on YPD plus on $2 \mathrm{mM}$ As (V) Figure 3C). Therefore, Pho4 increased its expression upon As $(\mathrm{V})$ addition but not $\mathrm{P}_{\mathrm{i}}$. This increase was higher when there was no phosphate in the medium. These results indicate that, despite their structural similarity, the cells sense differently arsenate and phosphate which, in turns, results in a different Pho4 expression.

\section{Pho4 IS INVOLVED IN THE RESPONSE TO ARSENITE}

We also tested the role of Pho4 in the response to arsenite via drop test analyses performed on plates supplemented with 2 and $3 \mathrm{mM}$ As (III). Similarly to the behavior on As (V) plates, the pho4 mutant was sensitive to sodium arsenite, while $\mathrm{PHO} 4^{\text {reint }}$ reverted the sensitive phenotype (Figure 5A). An electrophoretic shift was also observed in Pho4-myc mobility within $5 \mathrm{~min}$ after $2 \mathrm{mM}$ As (III) addition (Figure 5B).

When the expression of Pho4 was analyzed upon $2 \mathrm{mM}$ As (III) exposure, no induction of expression was detected $1 \mathrm{~h}$ after As (III) challenge in the wild type strain (Figure 5C). A statistically significant expression decrease was observed in the hogl mutant at $1 \mathrm{~h}$ of treatment. These data demonstrate that Pho 4 is also involved in the response to arsenite, although differently compare to arsenate. C. albicans cells therefore, differentiate between As (V) and As (III) compounds.

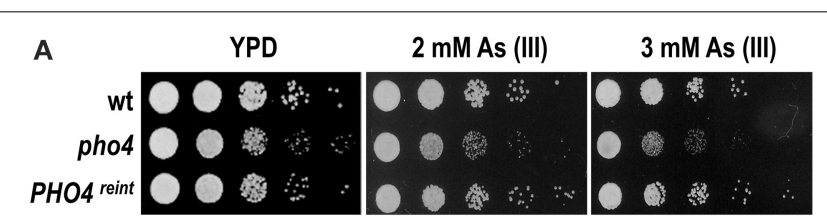

B

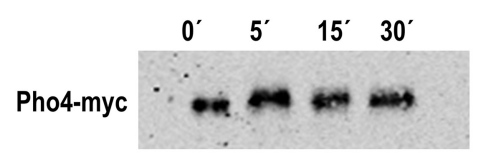

C

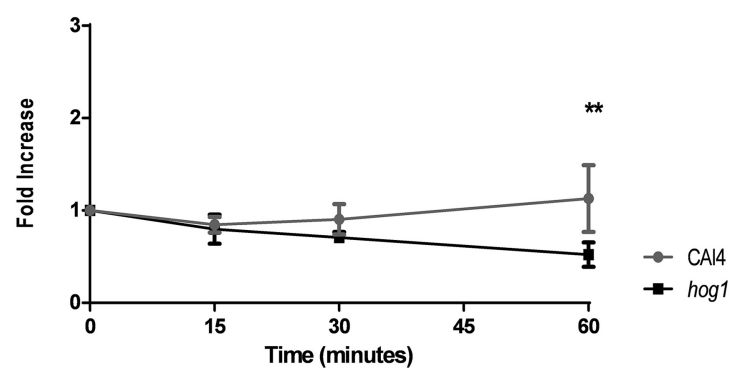

FIGURE 5 | Pho4 mediates the response to arsenite. (A) Ten-fold cell dilutions of the indicated strains were spotted on YPD plates supplemented with 2 and $3 \mathrm{mM}$ As (III). Plates were incubated at $37^{\circ} \mathrm{C}$ for $24 \mathrm{~h}$. (B) PHO4-reintegrant strain was challenged with 2 mM As (III). Pho4-myc was detected using anti-myc antibodies. (C) The PHO4 expression was quantified using the $P H O 4^{P R}$-CbLUC construction. CAF2 and hog 1 mutant carrying the gene reporter growing exponentially in YPD were challenge with $2 \mathrm{mM}$ As (III). Samples were taken at time indicated and luminescence quantified. Graph represent the mean of three independent experiments and the error bars is the standard deviation (SD). ${ }^{* *} p \leq 0.01$.

\section{ARSENITE TRIGGERS Hog1 PHOSPHORYLATION IN A Ssk1-INDEPENDENT WAY}

Lastly, we tested the role of the HOG pathway in the response to arsenite. Mutants defective in the HOG pathway were dropped on YPD plates supplemented with $3 \mathrm{mM}$ As (III) and incubated at $37^{\circ} \mathrm{C}$. Ssk1, Pbs2 and Hog1 defective mutants were sensitive to As (III) while no sensitivity was observed in the sko1 mutant; a hog1 sko1 double mutant was slightly more susceptible than single hog1 mutant indicating a possible role for Sko1 in As (III) tolerance (Figure 6A). As (III) susceptibility of mutants defective in the CWI pathway were also tested, no significant differences were detected compare to the parental strain (Supplementary Figure 5). This fact suggests that HOG pathway may be relevant for a proper response to As (III). The ability of As (III) to trigger Hog1 phosphorylation was tested. Hog1 became phosphorylated within $5 \mathrm{~min}$ after sodium arsenite addition and lasted for 15-30 min (Figure 6C). Remarkably, Hog1 phosphorylation was detected in an ssk1 mutant; although with a different kinetics, indicating that arsenite signaling is not fully Ssk1-dependent.

In order to determine the elements required for Hog 1 phosphorylation upon arsenite addition, others signaling defective mutants were analyzed. We checked mutants lacking elements of the MAPK module (Pbs2 and Ssk2) and mutants lacking upstream elements of the HOG pathway in C. albicans (Ssk1). We also analyzed elements that mediate the HOG pathway in S. cerevisiae such as Sho1, Msb2, and Opy2 that in C. albicans participate in the Cek1 pathway (Figure 6B). Exponentially growing cells were exposed to $2 \mathrm{mM}$ arsenite and samples were collected after $10 \mathrm{~min}$ (Figure 6D). Arsenite-induced-Hog1 phosphorylation depended on the MAPKK, Pbs2 and the MAPKKK, Ssk2. A sho1 ssk1 double mutant (as well as the sho1 ssk1 msb2 opy2 quadruple mutant) was able to trigger Hog1 phosphorylation similarly to wild type strain. Collectively, these data indicate that Hog1 phosphorylation upon arsenite challenge depends on the MAPK module of the pathway; however, the signal enters through an alternative way which is independent on Ssk1 and Sho1-Msb2Opy2. C. albicans, therefore senses and responds via a different mechanism to arsenate and arsenite.

\section{DISCUSSION}

Eukaryotic cells have developed different mechanisms to sense and respond to environmental changes. Among these mechanisms, MAPK pathways are crucial since they participate in vegetative cell growth, mating and the response to different kinds of stress. MAPK pathways are integrated by a core of three MAP kinases that activate each other by sequential phosphorylation. Signal coming from upstream elements triggers MAP kinase phosphorylation which, in fact, controls the transcriptional expression of specific genes through defined transcription factors. These signal transduction pathways are well conserved in all eukaryotic organisms (Kultz, 1998) and allow cells to respond and adapt to environmental alterations. C. albicans responds to stress mainly through the HOG pathway, although it does so with the cooperation of others MAPKs routes (Monge et al., 2006; Román et al., 2007). Hog1 becomes phosphorylated is response to osmotic, oxidative and metal-induced stress and 

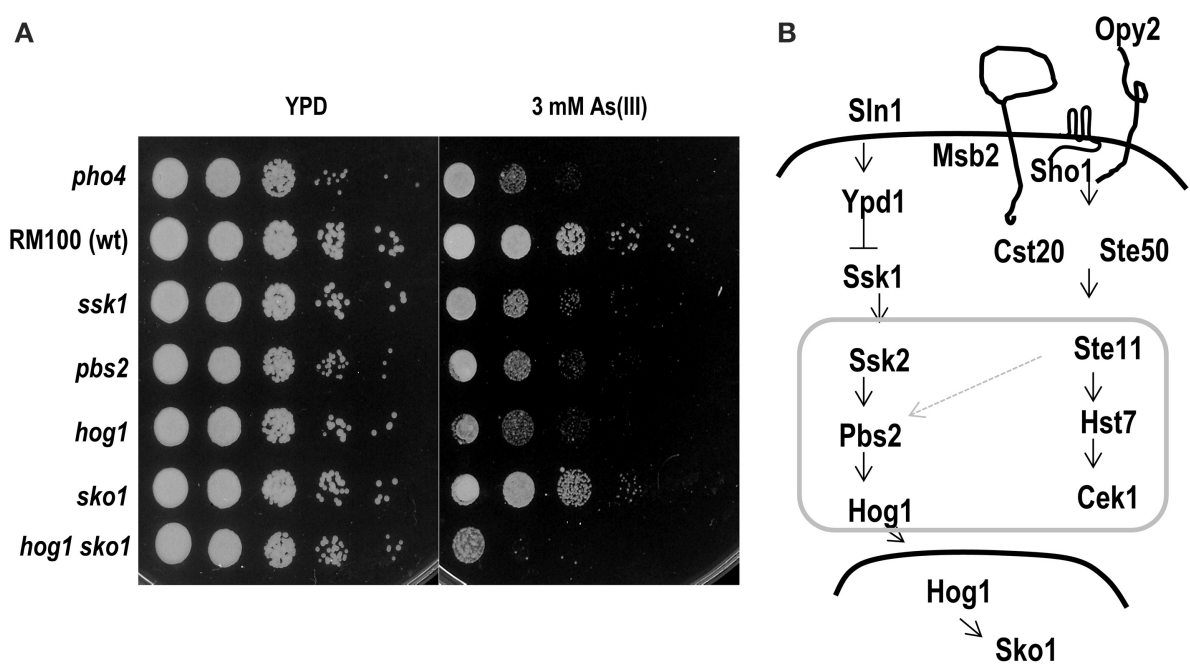

C

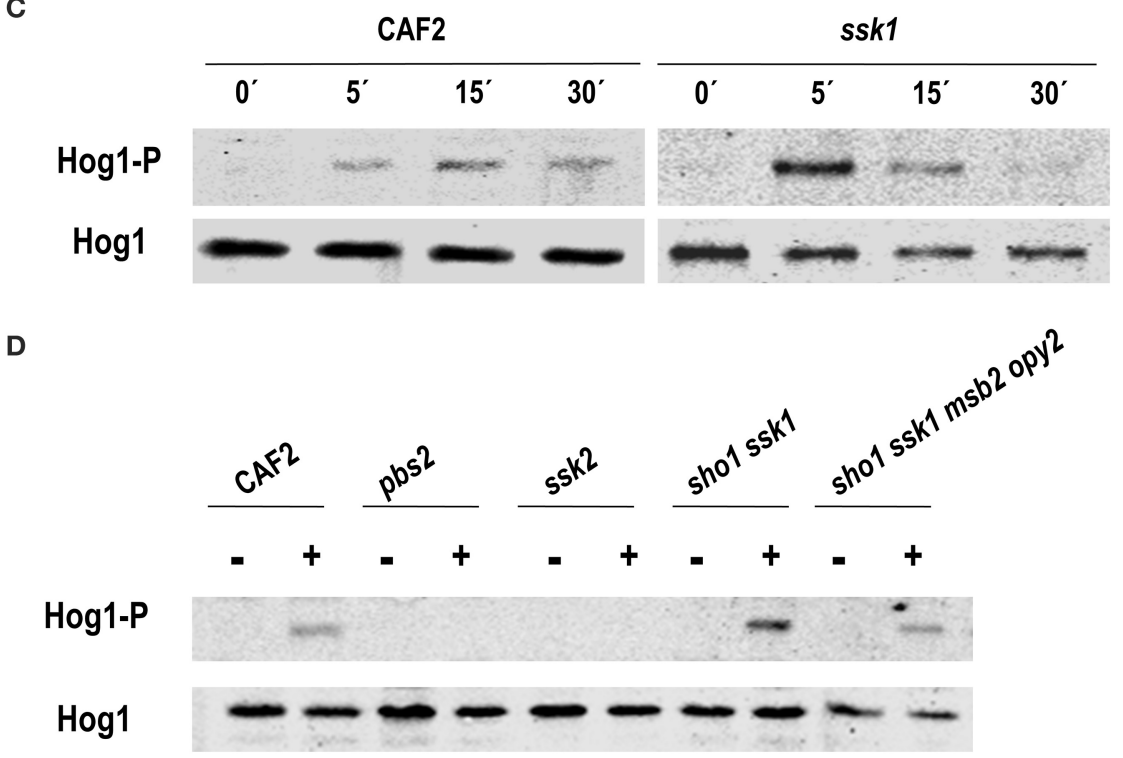

FIGURE 6 | Role of the HOG pathway in arsenite response. (A) Drop test of the indicated strains on YPD plates supplemented with $3 \mathrm{mM}$ As (III). Plates were incubated $24 \mathrm{~h}$ at $37^{\circ} \mathrm{C}$. (B) Schematic graph of HOG and Cek1-mediated pathways in $C$. albicans. A possible connection between both pathways is indicated with a light gray arrow. MAPK modules are framed within a gray square. Different $C$. albicans strains were challenged with $2 \mathrm{mM}$ As (III) at indicated time points (C) or at $10 \mathrm{~min}$ (D). Hog1 phosphorylation was detected using anti-p38-P and total amount of protein was detected with anti-Hog1 antibody. therefore, mutants defective in this pathway are sensitive to these kinds of stress (Alonso-Monge et al., 2003; Smith et al., 2004). Likewise, Hog1 becomes phosphorylated in response to As (III) and mutants in this pathway become sensitive to this compound.

It has been reported that As (III) (the most toxic form of arsenic) induces ROS production in mammals (Liu et al., 2001) and yeast (Menezes et al., 2008). In C. albicans the production of ROS by As (III) has not been demonstrated. Nevertheless, As (III) signaling differs from oxidative stress signaling. In fact, Ssk1 has been shown to be necessary to trigger Hog1 upon hydrogen peroxide (Chauhan et al., 2003), supporting the view that $C$. albicans HOG pathway, unlike $S$. cerevisiae, integrates a unique signaling branch (Cheetham et al., 2007) (Figure 6B). Our data indicate that As (III) triggers Hog1 phosphorylation in an
Ssk1-independent way. In S. cerevisiae, an Ssk1-independent Ssk2 activation upon osmotic challenge has been recently reported (Zhi et al., 2013). A similar alternative mechanism may explain Hog1 activation upon As (III) addition in C. albicans, since we observe Hog1 phosphorylation in $s s k 1$ but not in ssk2 and pbs2 mutants. Thus, Hogl phosphorylation requires elements of the MAPK module, but the signal comes through an element different to Ssk1 (Figure 7). This putative element does not belong to the transmembrane proteins previously involved in Cek1 phosphorylation: Sho1, Msb2, and Opy2 (Herrero-de-Dios et al., 2014) (Figure 6B) whose homologs in S. cerevisiae also mediate Hog1 phosphorylation (Tatebayashi et al., 2007; Ekiel et al., 2009). Another possibility is that As (III), after its uptake in the cell, generates intracellular oxidative stress which, in turn, triggers 


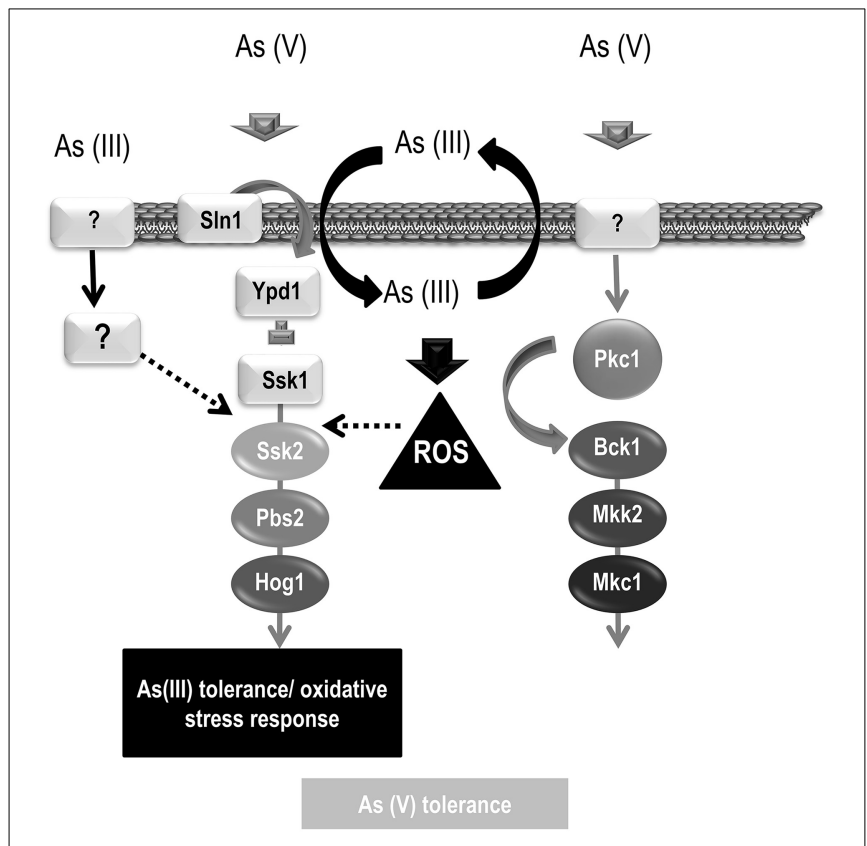

FIGURE 7 | Model of As (V) and As (III) signaling. A schematic representation of the MAPK pathways involved in arsenic signaling is depicted. As (V) triggers phosphorylation of both, HOG and CWI pathways. As (V) signaling requires Ssk1 suggesting that signal comes from outside the cell. Hog1 and Mkc1 phosphorylation may mediate transcription of genes involved in its detoxification or tolerance. As (III) triggers Hog1 phosphorylation and therefore, HOG pathway may control the transcription of genes involved in As (III) detoxification. As (III) signaling is independent of Ssk1 and others elements involves in Cek1 phosphorylation (Sho1, Opy2, and Msb2) but requires Ssk2 and Pbs2. The As (III) signaling may enter through an unknown element (represented as a question mark) or may enter the cells triggering Hog1 phosphorylation avoiding the three components system (SIn1-Ypd1-Ssk1).

Hog1 phosphorylation upstream Ssk2 in an Ssk1-independent way.

As (V) is sensed in a different way. In S. cerevisiae, As (V) triggers Slt2 (but not Hog1) phosphorylation (Matia-Gonzalez and Rodriguez-Gabriel, 2011). In this case, mutants in the CWI pathway are susceptible to arsenate while mutants defective in the HOG pathway are not (Matia-Gonzalez and Rodriguez-Gabriel, 2011). Unlike S. cerevisiae, As (V) induces Hogl and Mkcl phosphorylation in C. albicans but mutants in one or both pathways do not display enhanced susceptibility to arsenate on plate. The fact that CWI mutants do not show increased susceptibility to As (V) does not argue against its role in As (V) tolerance, as it has been already described that $\mathrm{Mkcl}$ is activated by oxidative stress but $m k c 1$ cells are not sensitive to oxidant such as hydrogen peroxide (Navarro-García et al., 2005). Hog1 phosphorylation upon As $(V)$ challenge requires Ssk1, suggesting that the signal is generated via the canonical membrane route, and presumably, outside to the cells (Figure 7). Ssk1 was also important to trigger Mkc1 phosphorylation in response to As $(\mathrm{V})$; this key role played by the HOG pathway in controlling $\mathrm{Mkcl}$ activation has been previously reported for hydrogen peroxide phosphorylation (Arana et al., 2005; Navarro-García et al., 2005) and demonstrate that
MAPK pathways work coordinately. Interestingly, As (V) is only able to trigger MAPKs phosphorylation in rich medium (YPD) and neither Hogl nor Mkcl phosphorylation was detected when cultures were grown in phosphate deficient medium (Figure 4C). This different response could be explained because fungal cells express high-affinity phosphate transporters when phosphate is not available in surrounding environment. Phosphate starvation induces the phosphate signaling and response ( $\mathrm{PHO}$ ) pathway which controls the expression of specific genes such as the highaffinity phosphate transporter Pho84 (reviewed by Tomar and Sinha, 2014). This and others transporters/sensors with a higher affinity could be responsible for differentiating between phosphate and arsenate in spite of their structural similarity. In this phosphate starving environment, addition of inorganic phosphate triggered Hog1 and Cek1 phosphorylation. Cek1 become activated when cells resume growth from stationary phase to fresh medium (Román et al., 2005). Under our experimental conditions (a phosphate challenge in a phosphate deficient medium), Cek1 phosphorylation could be explained as a growth initiation signal after a starvation period. The expression of the high-affinity phosphate transporter Pho84 has been shown to be down-regulated in a hog1 mutant in YPDAU medium (Enjalbert et al., 2006) indicating that the HOG pathway contributes, either directly or indirectly, to control its expression. Our work shows that Hog1 senses the presence of phosphate when environmental phosphate concentration is low. Therefore, it may contribute to phosphate homeostasis, presumably mediating Pho84 expression among others required proteins. The transcription factor involved in this regulation remains unknown and it is tempting to speculate that Pho4 could be a downstream Hog1-target. Our data do not demonstrate a direct connection between the HOG and $\mathrm{PHO}$ pathways, but suggest a relationship between both signaling mechanisms. A constitutive $\mathrm{PHO} 4$ expression $\left(\mathrm{PHO}^{\text {reint }}\right)$ leads to an increased Hogl basal phosphorylation while pho4 mutants display a Hogl altered phosphorylation pattern. These facts indicate that the intracellular concentration of Pho4 has to be tightly controlled, being otherwise a stress signal (phosphorylating Hog1) in Candida cells. On the other hand, the absence of Hogl does not impair Pho4 expression upon As (V) challenge; but it does so upon As (III) addition.

Pho4 is required for growth in medium lacking phosphate in C. albicans (Figure 4A) (Homann et al., 2009; Romanowski et al., 2012). In S. cerevisiae Pho4 function depends on phosphate availability regulating its dynamic and phosphorylation (Komeili and O'Shea, 1999). When the phosphate concentration is high in the medium, Pho4 becomes hyper-phosphorylated by the Pho80-Pho85 complex which results in Pho4 being excluded from the nucleus; when the environmental phosphate concentration is low Pho81 represses the Pho80-Pho85 complex leading to Pho4 hypo-phosphorylation and Pho4 remains in the nucleus inducing the expression of high-affinity transporters (Pho84 and Pho89) and secretory phosphatases (Pho5, Pho11, and Pho12). A similar mechanism can be suggested for C. albicans since a delay in electrophoretic Pho4 mobility, probably due to Pho4 phosphorylation, was detected when a culture was shifted from a medium depleted in phosphate to normal phosphate availability. 
In addition to the role of Pho4 in phosphate metabolism, it is also involved in As (V) tolerance in C. albicans. This role can be explained because As (V) is structurally homolog to inorganic phosphate $\left(\mathrm{P}_{\mathrm{i}}\right)$. As $(\mathrm{V})$ also induces a delay in the electrophoretic mobility which suggests again phosphorylation. This putative phosphorylation(s) must switch off the transcription of highaffinity transporters and secretory phosphatases, thus avoiding the uptake of phosphate (or, in this case, environmental arsenate). It is also possible that Pho4 becomes phosphorylated at different sites upon Pi and As (V) addition, inducing specific and separated responses, since $C$. albicans can discriminate between $\mathrm{Pi}$ and As (V). This discrimination was observed in the signaling but also in Pho4 expression. As (V) induces Pho4 transcriptional activation (higher when environmental phosphate concentration is low) although phosphate starvation did not affect Pho4 expression (Figure 4B). This transcriptional induction is short in time and may correlate with the induction of an As (V) specific response.

Pho4 plays an additional role in As (III) tolerance. As (V) enters the cells through phosphate transporter and then, is reduced to As (III) by reductases. It is not surprising that mechanisms involved in As (V) tolerance could be implicated in As (III) adaptation as well. In this sense, Cap1 was identified in our screening searching for mutants sensitive to As (V). The homolog to Cap1 in S. cerevisiae, Yap1 controls As (III) response in cooperation with Met4 (Thorsen et al., 2007). Others transcription factors identified as implicated in As (V) tolerance in C. albicans were Ada2, Wor3, and orf19.2260. Zhou and co-workers showed that the $S$. cerevisiae ada 2 mutant was susceptible to arsenite in a genome-wide screening (Zhou et al., 2009). Our work, link these transcription factors to As (V) tolerance.

Why are mechanisms mediating As (III) and As (V) tolerance still functional in a non-environmental yeast? Although speculative, it is clear that humans are being constantly exposed to inorganic arsenic through food and drinking water causing different disorders (Flora, 2011). Intestinal microbiome plays an important role in arsenic detoxification in order to remove it from the body (Rowland, 1981; Lu et al., 2013). C. albicans belong to human gut microbiome and therefore it must promote As (and others chemicals) detoxification. Therefore, evolution may have well played a role in the maintenance and tuning of these mechanisms to cope with toxic arsenic compounds. In this context, coordinating MAPK signaling with the PHO pathways is an attractive mechanism that may be useful for adaptation to the commensal state.

\section{ACKNOWLEDGMENTS}

Work in our laboratory is supported by Grants S2010/BMD24 (from Comunidad de Madrid), BIO2012-31839 and PIM2010EPA-00658 (from Ministerio de Educación y Ciencia). We thank D. Sanglard for providing the Transcription Factor Knock-out library and J. Quinn for providing the ssk2 strain. We also thank R. Sanabrias and B. Heranz for their practical assistance and B. Eisman for the initial contribution to the work.

\section{SUPPLEMENTARY MATERIAL}

The Supplementary Material for this article can be found online at: http://www.frontiersin.org/journal/10.3389/fmicb.2015. 00118/abstract
Supplementary Figure 1 | Sequences of $C b L U C$ gene (A) and protein (B) adapted for $\boldsymbol{C}$. albicans. Accession number: KP202872.

Supplementary Figure 2 | Sensitivity to arsenate displayed by transcription factor knock-out mutants. Transcription factor mutants identified as sensitive to arsenate were spotted on YPD and YPD plus $2 \mathrm{mM}$ arsenate and incubated at $37^{\circ} \mathrm{C}$ for $24 \mathrm{~h}$.

Supplementary Figure 3 | Comparison of ScPho4 and CaPho4 sequences. $\mathrm{ScPho} 4$ and $\mathrm{CaPho} 4$ were aligned using the default setting of Clustaw2. Dark arrows sign ScPho4 reported phosphorylation sites (Sp1, SP2, SP3, SP4, and SP6) while gray arrows indicate putative phosphorylation sites detected in $\mathrm{CaPho} 4$ sequence.

Supplementary Figure $4 \mid P_{i}$ and $A s(V)$ trigger different signaling pathways. A wild type strain (CAF 2) grown exponentially in SD medium was split in two and $2 \mathrm{mM}$ Pi or $2 \mathrm{mM}$ As (V) was added to each culture. Samples were taken at different time points and MAP kinase phosphorylation was analyzed using specific antibodies. Hog1-P, Cek1-P, and Mkc1-P designate the phosphorylated form of the MAPKs while Hog1 indicates the total Hog1 protein.

Supplementary Figure 5 | Defective mutants in the CWI pathway are not susceptible to As (III). Ten-fold dilutions of the indicated strains were spotted on YPD and YPD plus $3 \mathrm{mM}$ As (III). Plates were incubated at $37^{\circ} \mathrm{C}$ for $24 \mathrm{~h}$.

\section{REFERENCES}

Alonso-Monge, R., Navarro-García, F., Román, E., Negredo, A. I., Eisman, B., Nombela, C., et al. (2003). The Hogl mitogen-activated protein kinase is essential in the oxidative stress response and chlamydospore formation in Candida albicans. Eukaryot. Cell 2, 351-361. doi: 10.1128/EC.2.2.351-361.2003

Alonso-Monge, R., Román, E., Arana, D. M., Prieto, D., Urrialde, V., Nombela, C., et al. (2010). The Skol protein represses the yeast-to-hypha transition and regulates the oxidative stress response in Candida albicans. Fungal. Genet. Biol. 47, 587-601. doi: 10.1016/j.fgb.2010.03.009

Arana, D. M., Alonso-Monge, R., Du, C., Calderone, R., and Pla, J. (2007). Differential susceptibility of mitogen-activated protein kinase pathway mutants to oxidative-mediated killing by phagocytes in the fungal pathogen Candida albicans. Cell Microbiol. 9, 1647-1659. doi: 10.1111/j.1462-5822.2007.00898.x

Arana, D. M., Nombela, C., Alonso-Monge, R., and Pla, J. (2005). The Pbs2 MAP kinase kinase is essential for the oxidative-stress response in the fungal pathogen Candida albicans. Microbiology 151, 1033-1049. doi: 10.1099/mic.0.27723-0

Berdicevsky, I., Duek, L., Merzbach, D., and Yannai, S. (1993). Susceptibility of different yeast species to environmental toxic metals. Environ. Pollut. 80, 41-44. doi: 10.1016/0269-7491(93)90007-B

Bienert, G. P., Schussler, M. D., and Jahn, T. P. (2008). Metalloids: essential, beneficial or toxic? Major intrinsic proteins sort it out. Trends Biochem. Sci. 33, 20-26. doi: 10.1016/j.tibs.2007.10.004

Calera, J. A., Zhao, X. J., and Calderone, R. (2000). Defective hyphal development and avirulence caused by a deletion of the SSK1 response regulator gene in Candida albicans. Infect. Immun. 68, 518-525. doi: 10.1128/IAI.68.2.518525.2000

Chauhan, N., Inglis, D., Román, E., Pla, J., Li, D., Calera, J. A., et al. (2003). Candida albicans response regulator gene SSK1 regulates a subset of genes whose functions are associated with cell wall biosynthesis and adaptation to oxidative stress. Eukaryot. Cell 2, 1018-1024. doi: 10.1128/EC.2.5.1018-1024.2003

Cheetham, J., Smith, D. A., da Silva, D. A., Doris, K. S., Patterson, M. J., Bruce, C. R., et al. (2007). A single MAPKKK regulates the Hogl MAPK pathway in the pathogenic fungus Candida albicans. Mol. Biol. Cell 18, 4603-4614. doi: 10.1091/mbc.E07-06-0581

Ekiel, I., Sulea, T., Jansen, G., Kowalik, M., Minailiuc, O., Cheng, J., et al. (2009) Binding the atypical RA domain of Ste50p to the unfolded Opy2p cytoplasmic tail is essential for the high-osmolarity glycerol pathway. Mol. Biol. Cell 20, 5117-5126. doi: 10.1091/mbc.E09-07-0645

Enjalbert, B., Smith, D. A., Cornell, M. J., Alam, I., Nicholls, S., Brown, A. J., et al. (2006). Role of the Hogl stress-activated protein kinase in the global 
transcriptional response to stress in the fungal pathogen Candida albicans. Mol. Biol. Cell 17, 1018-1032. doi: 10.1091/mbc.E05-06-0501

Flora, S. J. (2011). Arsenic-induced oxidative stress and its reversibility. Free Radic. Biol. Med. 51, 257-281. doi: 10.1016/j.freeradbiomed.2011.04.008

Fonzi, W. A., and Irwin, M. Y. (1993). Isogenic strain construction and gene mapping in Candida albicans. Genetics 134, 717-728.

Herrero-de-Dios, C., Alonso-Monge, R., and Pla, J. (2014). The lack of upstream elements of the Cek1 and Hog1 mediated pathways leads to a synthetic lethal phenotype upon osmotic stress in Candida albicans. Fungal. Genet. Biol. 69C, 31-42. doi: 10.1016/j.fgb.2014.05.010

Homann, O. R., Dea, J., Noble, S. M., and Johnson, A. D. (2009). A phenotypic profile of the Candida albicans regulatory network. PLoS. Genet. 5:e1000783. doi: 10.1371/journal.pgen.1000783

Köhler, G. A., White, T. C., and Agabian, N. (1997). Overexpression of a cloned IMP dehydrogenase gene of Candida albicans confers resistance to the specific inhibitor mycophenolic acid. J. Bacteriol. 179, 2331-2338.

Komeili, A., and O'Shea, E. K. (1999). Roles of phosphorylation sites in regulating activity of the transcription factor Pho4. Science 284, 977-980. doi: 10.1126/science.284.5416.977

Kultz, D. (1998). Phylogenetic and functional classification of mitogen- and stress-activated protein kinases. J. Mol. Evol. 46, 571-588. doi: 10.1007/PL000 06338

Levin, D. E. (2011). Regulation of cell wall biogenesis in Saccharomyces cerevisiae: the cell wall integrity signaling pathway. Genetics 189, 1145-1175. doi: 10.1534/genetics.111.128264

Liu, S. X., Athar, M., Lippai, I., Waldren, C., and Hei, T. K. (2001). Induction of oxyradicals by arsenic: implication for mechanism of genotoxicity. Proc. Natl. Acad. Sci. U.S.A. 98, 1643-1648. doi: 10.1073/pnas.98.4.1643

Liu, Z., Boles, E., and Rosen, B. P. (2004). Arsenic trioxide uptake by hexose permeases in Saccharomyces cerevisiae. J. Biol. Chem. 279, 17312-17318. doi: 10.1074/jbc.M314006200

Lu, K., Cable, P. H., Abo, R. P., Ru, H., Graffam, M. E., Schlieper, K. A., et al. (2013). Gut microbiome perturbations induced by bacterial infection affect arsenic biotransformation. Chem. Res. Toxicol. 26, 1893-1903. doi: 10.1021/ tx4002868

Maciaszczyk-Dziubinska, E., Migdal, I., Migocka, M., Bocer, T., and Wysocki, R. (2010). The yeast aquaglyceroporin Fpslp is a bidirectional arsenite channel. FEBS Lett. 584, 726-732. doi: 10.1016/j.febslet.2009.12.027

Martín, H., Arroyo, J., Sánchez, M., Molina, M., and Nombela, C. (1993). Activity of the yeast MAP kinase homologue Slt2 is critically required for cell integrity at 37 degrees C. Mol. Gen. Genet. 241, 177-184. doi: 10.1007/BF00280215

Matia-Gonzalez, A. M., and Rodriguez-Gabriel, M. A. (2011). Slt2 MAPK pathway is essential for cell integrity in the presence of arsenate. Yeast 28, 9-17. doi: $10.1002 /$ yea. 1816

Menezes, R. A., Amaral, C., Batista-Nascimento, L., Santos, C., Ferreira, R. B., Devaux, F., et al. (2008). Contribution of Yap1 towards Saccharomyces cerevisiae adaptation to arsenic-mediated oxidative stress. Biochem. J. 414, 301-311. doi: 10.1042/BJ20071537

Migdal, I., Ilina, Y., Tamas, M. J., and Wysocki, R. (2008). Mitogen-activated protein kinase $\operatorname{Hog} 1$ mediates adaptation to G1 checkpoint arrest during arsenite and hyperosmotic stress. Eukaryot. Cell 7, 1309-1317. doi: 10.1128/EC. 00038-08

Monge, R. A., Roman, E., Nombela, C., and Pla, J. (2006). The MAP kinase signal transduction network in Candida albicans. Microbiology 152, 905-912. doi: $10.1099 /$ mic. $0.28616-0$

Navarro-García, F., Eisman, B., Fiuza, S. M., Nombela, C., and Pla, J. (2005). The MAP kinase Mkclp is activated under different stress conditions in Candida albicans. Microbiology 151, 2737-2749. doi: 10.1099/mic.0.28038-0

Navarro-García, F., Sánchez, M., Pla, J., and Nombela, C. (1995). Functional characterization of the $M K C 1$ gene of Candida albicans, which encodes a mitogen-activated protein kinase homolog related to cell integrity. Mol. Cell Biol. 15, 2197-2206.

Negredo, A., Monteoliva, L., Gil, C., Pla, J., and Nombela, C. (1997), Cloning, analysis and one-step disruption of the ARG5,6 gene of Candida albicans. Microbiology 143, 297-302. doi: 10.1099/00221287143-2-297

Prieto, D., Roman, E., Correia, I., and Pla, J. (2014). The HOG pathway is critical for the colonization of the mouse gastrointestinal tract by Candida albicans. PLoS ONE 9:e87128. doi: 10.1371/journal.pone.0087128
Rauceo, J. M., Blankenship, J. R., Fanning, S., Hamaker, J. J., Deneault, J. S., Smith, F. J., et al. (2008). Regulation of the Candida albicans cell wall damage response by transcription factor Sko1 and PAS kinase Psk1. Mol. Biol. Cell 19, 2741-2751. doi: 10.1091/mbc.E08-02-0191

Román, E., Arana, D. M., Nombela, C., Alonso-Monge, R., and Pla, J. (2007). MAP kinase pathways as regulators of fungal virulence. Trends Microbiol. 15, 181-190. doi: 10.1016/j.tim.2007.02.001

Román, E., Nombela, C., and Pla, J. (2005). The Shol adaptor protein links oxidative stress to morphogenesis and cell wall biosynthesis in the fungal pathogen Candida albicans. Mol. Cell Biol. 25, 10611-10627. doi: 10.1128/MCB.25.23.10611-10627.2005

Romanowski, K., Zaborin, A., Valuckaite, V., Rolfes, R. J., Babrowski, T., Bethel, C., et al. (2012). Candida albicans isolates from the gut of critically ill patients respond to phosphate limitation by expressing filaments and a lethal phenotype. PLoS ONE 7:e30119. doi: 10.1371/journal.pone.0030119

Rosen, B. P. (2002). Biochemistry of arsenic detoxification. FEBS Lett. 529, 86-92. doi: 10.1016/S0014-5793(02)03186-1

Rowland, I. (1981). The influence of the gut microflora on food toxicity. Proc. Nutr. Soc. 40, 67-74. doi: 10.1079/PNS19810011

San José, C., Monge, R. A., Perez-Díaz, R., Pla, J., and Nombela, C. (1996). The mitogen-activated protein kinase homolog HOG1 gene controls glycerol accumulation in the pathogenic fungus Candida albicans. J. Bacteriol. 178, 5850-5852.

Smith, D. A., Nicholls, S., Morgan, B. A., Brown, A. J., and Quinn, J. (2004). A conserved stress-activated protein kinase regulates a core stress response in the human pathogen Candida albicans. Mol. Biol. Cell 15, 4179-4190. doi: 10.1091/mbc.E04-03-0181

Sotelo, J., and Rodriguez-Gabriel, M. A. (2006). Mitogen-activated protein kinase Hogl is essential for the response to arsenite in Saccharomyces cerevisiae. Eukaryot. Cell 5, 1826-1830. doi: 10.1128/EC.00225-06

Tamas, M. J., and Wysocki, R. (2001). Mechanisms involved in metalloid transport and tolerance acquisition. Curr. Genet. 40, 2-12. doi: 10.1007/s002940 100234

Tatebayashi, K., Tanaka, K., Yang, H. Y., Yamamoto, K., Matsushita, Y., Tomida, T., et al. (2007). Transmembrane mucins Hkr1 and Msb2 are putative osmosensors in the SHO1 branch of yeast HOG pathway. EMBO J. 26, 3521-3533. doi: 10.1038/sj.emboj.7601796

Thorsen, M., Di, Y., Tangemo, C., Morillas, M., Ahmadpour, D., Van der Does, C., et al. (2006). The MAPK Hoglp modulates Fpslp-dependent arsenite uptake and tolerance in yeast. Mol. Biol. Cell 17, 4400-4410. doi: 10.1091/mbc.E0604-0315

Thorsen, M., Lagniel, G., Kristiansson, E., Junot, C., Nerman, O., Labarre, J., et al. (2007). Quantitative transcriptome, proteome, and sulfur metabolite profiling of the Saccharomyces cerevisiae response to arsenite. Physiol. Genomics 30, 35-43. doi: 10.1152/physiolgenomics.00236.2006

Tomar, P., and Sinha, H. (2014). Conservation of PHO pathway in ascomycetes and the role of Pho84. J. Biosci. 39, 525-536. doi: 10.1007/s12038-0149435-y

Vandeputte, P., Pradervand, S., Ischer, F., Coste, A. T., Ferrari, S., Harshman, K., et al. (2012). Identification and functional characterization of Rcal, a transcription factor involved in both antifungal susceptibility and host response in Candida albicans. Eukaryot. Cell 11, 916-931. doi: 10.1128/EC.00134-12

Wysocki, R., Bobrowicz, P., and Ulaszewski, S. (1997). The Saccharomyces cerevisiae $A C R 3$ gene encodes a putative membrane protein involved in arsenite transport. J. Biol. Chem. 272, 30061-30066. doi: 10.1074/jbc.272.48.30061

Wysocki, R., Chery, C. C., Wawrzycka, D., Van, H. M., Cornelis, R., Thevelein, J. M., et al. (2001). The glycerol channel Fpslp mediates the uptake of arsenite and antimonite in Saccharomyces cerevisiae. Mol. Microbiol. 40, 1391-1401. doi: 10.1046/j.1365-2958.2001.02485.x

Wysocki, R., and Tamas, M. J. (2010). How Saccharomyces cerevisiae copes with toxic metals and metalloids. FEMS Microbiol. Rev. 34, 925-951. doi 10.1111/j.1574-6976.2010.00217.x

Zhi, H., Tang, L., Xia, Y., and Zhang, J. (2013). Ssklp-independent activation of Ssk2p plays an important role in the osmotic stress response in Saccharomyces cerevisiae: alternative activation of Ssk $2 p$ in osmotic stress. PLoS ONE 8:e54867. doi: 10.1371/journal.pone.0054867

Zhou, X., Arita, A., Ellen, T. P., Liu, X., Bai, J., Rooney, J. P., et al. (2009). A genomewide screen in Saccharomyces cerevisiae reveals pathways affected by arsenic toxicity. Genomics 94, 294-307. doi: 10.1016/j.ygeno.2009.07.003 
Conflict of Interest Statement: The authors declare that the research was conducted in the absence of any commercial or financial relationships that could be construed as a potential conflict of interest.

Received: 26 November 2014; accepted: 29 January 2015; published online: 11 February 2015.

Citation: Urrialde V, Prieto D, Pla J and Alonso-Monge R (2015) The Pho4 transcription factor mediates the response to arsenate and arsenite in Candida albicans. Front. Microbiol. 6:118. doi: 10.3389/fmicb.2015.00118
This article was submitted to Fungi and Their Interactions, a section of the journal Frontiers in Microbiology.

Copyright (C) 2015 Urrialde, Prieto, Pla and Alonso-Monge. This is an openaccess article distributed under the terms of the Creative Commons Attribution License (CC BY). The use, distribution or reproduction in other forums is permitted, provided the original author(s) or licensor are credited and that the original publication in this journal is cited, in accordance with accepted academic practice. No use, distribution or reproduction is permitted which does not comply with these terms. 